\title{
Chemical Pneumonia Due to Paint Thinner Ingestion: A Case Report and Literature Review
}

\author{
(D) Mehmet Koçak¹, (D) Kurtuluș Açıksarı²
}

${ }^{1}$ Clinic of Emergency Medicine, Fatih Sultan Mehmet Training and Research Hospital, İstanbul, Turkey

2Department of Emergency Medicine, İstanbul Medeniyet University Faculty of Medicine, İstanbul, Turkey

\begin{abstract}
Paint thinner is an organic solvent which includes aromatic hydrocarbons and is widely used in the paint, varnish, and plastic products industries. Regular inhalation of such solvents is known to cause chronic intoxication. Oral thinner over-exposure is rare but quite fatal. Volatile liquids can cause pulmonary complications even with oral ingestion. Therefore, in patients with oral volatile liquid thinner intake, emergency physicians should be aware of local complications as well as the systematic effect even with initial normal physical or radiological findings. In this report, we present a patient with chemical pneumonia due to self-vomiting after accidentally drinking paint thinner.
\end{abstract}

Keywords: Chemical pneumonitis, thinner, hydrocarbons, ingestion

\section{Introduction}

Exposure to hydrocarbons is common in modern society. Thinner is an organic solvent that contains aromatic hydrocarbons such as toluene and xylene and is easily accessible in products such as gasoline, paint, varnish, and plastic production industries. Types of exposure include unintentional ingestion, intentional recreational abuse, unintentional inhalation, and dermal exposure or oral ingestion in a suicide attempt. Regular inhalation of such solvents is known to cause chronic intoxication. Toluene and xylene are absorbed in the gastrointestinal tract and are shown to cause rhabdomyolysis, metabolic acidosis, hepatic and renal dysfunction, neurotoxicity, and pulmonary toxicity due to their lipophilic affinity in rodents and humans.

Oral thinner over-exposure is rare, but quite fatal. Oral intake of 45-50 mL thinner is known to cause severe complications (1). Toluene accumulates in the liver following oral exposure, and thinner accumulates in the brain following respiratory exposure. Volatile organic compounds act as central nervous system depressors due to their lipophilic characteristics and cause death after oral ingestion. Herein we report an adult male who was admitted to our hospital with complaint of fever with the history of paint thinner ingestion.

\section{Case Report}

A 54-year-old male patient presented to the emergency department (ED) with fever. He had drunk half bottle of paint thinner (approximately $100 \mathrm{~mL}$ ) accidentally 9 hours before his admission. Upon realizing his mistake, he had self-induced vomiting in an attempt to get rid of the thinner about 2-3 times, and then had drunk a lot of water to minimize the effect of the thinner.

Physical examination revealed an alert and oriented male with a regular pulse of 110 beats/min, blood pressure of 119/75 mmHg, respiratory rate of 20 breaths/min, body temperature of 38.9 ${ }^{\circ} \mathrm{C}$, and $\mathrm{O}_{2}$ saturation of $98 \%$ on room air. Cardiac examination revealed normal $\$ 1$ and $\$ 2$ with no audible murmurs, rubs, or gallops. Chest examination showed bilateral equal aeration; there were crepitant rales over the right lower zone but no wheezing or rhonchi. The abdomen was soft, non-tender, and non-distended, without any evidence of organomegaly. No lower 


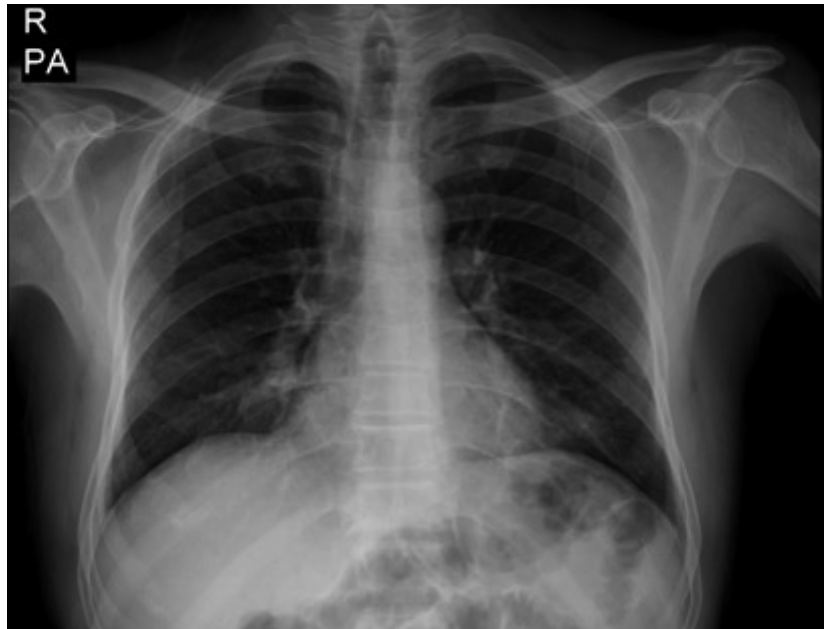

Figure 1. Normal posterior-anterior chest X-ray without anysign of pneumonia taken in the first hour of the admission

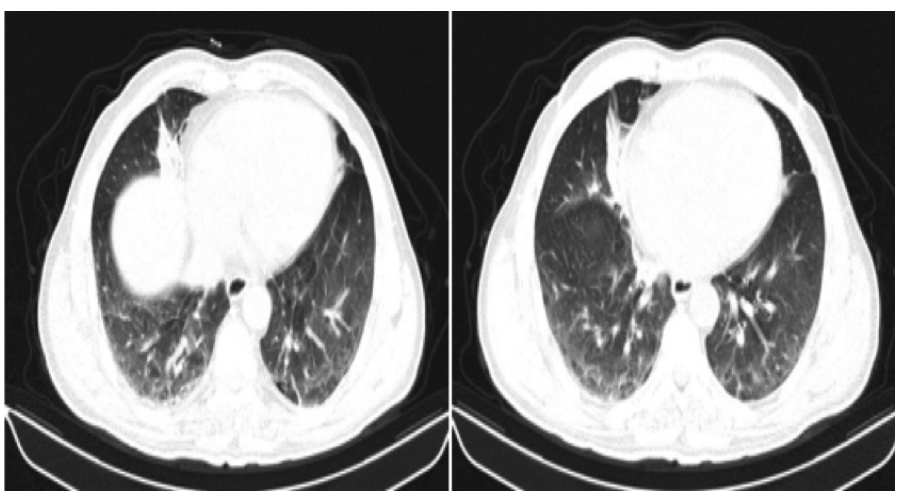

Figure 2. Chest computed tomography scan (without contrast) performed on the $16^{\text {th }}$ hour of ingestion: Consolidation are as containing air bronchograms secondary to parenchymal infiltration can be seen in the anterobasal segment of inferior lobe of the right lung

extremity edema, cyanosis, or clubbing was seen. The peripheral arterial pulses were palpable. The neurologic examination was normal.

Laboratory tests, including arterial blood gas, renal and liver function tests, and a comprehensive metabolic panel were all within normal limits. The results of the complete blood count were as follows; white blood cell count: 19.300 L; neutrophil ratio: $83.4 \%$; platelet count, hemoglobin and hematocrit levels were within the normal range. No invasive or infiltrative lesion was detected in the chest X-ray (Figure 1).

The patient was hospitalized for supportive treatment and further workup and monitoring. At third hour of admission, the patient developed an attack of bronchospasm. Meanwhile, the vital findings were as follows: $\mathrm{SpO}_{2}$ : 97\% (on room air), pulse: 109/min, blood pressure: 120/67 mmHg. After inhalation of nebulized ipratropium bromide plus budesonide, his symptoms regressed. At $7^{\text {th }}$ hour of admission, the same inhaler treatment was administered because of a recurrent attack of bronchospasm. Therefore, chest computed tomography (CT) scan (without contrast) was performed for differential diagnosis. It showed consolidation areas containing air bronchograms secondary to parenchymal infiltration in the inferior lobe of the right lung (Figure 2). Hence, chemical pneumonitis was diagnosed and amoxicillin-clavulanate was started. Patient was discharged after he had been symptom-free and hemodynamically stable for 24 hours on the $3^{\text {rd }}$ day of admission.

\section{Discussion}

Oral intake or inhalation of less than $1 \mathrm{~mL}$ of some hydrocarbons has been shown to cause chemical pneumonia and death. Respiratory symptoms generally begin in the first few hours after exposure and usually resolve in 2-8 days. Symptoms such as cough and broncho-obstruction may occur shortly after oral intake. Tachypnea, wheezing, and chemical pneumonitis may follow thereafter. In such cases death due to chemical exposure, which is usually related to bacterial infections and other respiratory complications, may ensue (2). Our patient, who had self-vomited after accidentally having drunk thinner, developed fever and respiratory signs. Inducing vomiting in such patients is not advised because it can increase the risk of pulmonary complications as a result of aspiration (3).

Hydrocarbons can be aspirated after oral ingestion and may cause toxic effects on lungs. Nonetheless, they may also cause systematic toxic effects due to oral, respiratory, or dermal exposure. Especially in thinner and naphtha intoxications respiratory symptoms can slowly occur. Death has been reported in patients with multi-organ failure secondary to oral intake of hydrocarbons (4). While signs of chemical pneumonitis can appear in the chest radiogram as early as 30 minutes after exposure to any chemical in symptomatic patients, they may variably occur between 2-24 hours in asymptomatic patients (5). In our case, fever and leukocytosis with negative chest X-ray occurred 9 hours after the oral intake and signs of chemical pneumonitis in the chest CT settled 16 hours after the intake. Therefore, the patient was given broad-spectrum antibiotic due to increasing infiltrate in radiological imaging. Occurrence and persistence of fever, increasing infiltrate in chest radiograph, leukocytosis or sputum or tracheal aspirate positive for bacteria after hydrocarbon aspiration over 48 hours suggests bacterial superinfection. Hence, pneumonitis caused by hydrocarbon aspiration should not be treated routinely with antibiotics unless signs of secondary infection (6).

In the literature reports fatal toluene levels were reported to be 29-119 $\mu \mathrm{g} / \mathrm{g}$ in 3 cases (7); in one case who was externalized in 
full health after 8 days of follow-up in the intensive care unit, toluene level was reported to be $17 \mu \mathrm{g} / \mathrm{g}$ (1). Blood toluene level test cannot be performed in our hospital therefore, we cannot provide any data about the blood toluene level of our case.

In a report of 20 cases with toluene inhalation, the most frequent complaint was muscle weakness due to hypokalemia, followed by altered mental status and gastrointestinal complaints including nausea, vomiting, and abdominal pain with elevation of creatinephosphokinase concentration to the level approximately 6 times the upper normal limit and elevated gamma-glutamyl transpeptidase and alkaline phosphatase (ALP) levels (8). In another report of 37 adult thinner intoxication cases, the most frequent complaint was nausea and vomiting with elevated ALP and lactate dehydrogenase levels, approximately 2-3 times the upper limit of normal (9). In our case, there was no abnormality in the electrolytes, or liver, or kidney function tests.

Treatment with surfactant in pediatric patients with acute respiratory distress syndrome (ARDS) due to toluene inhalation and treatment with budesonide and nitric oxide in patients with respiratory distress and bronchospasm were found effective (5). On the contrary, treatment with corticosteroids in dogs with fulminant hydrocarbon aspiration was found ineffective (10). Our patient had fever without respiratory distress in initial examination and had bouts of bronchospasm 12 hours and 16 hours after exposure. The patient was treated with inhalation of nebulized ipratropium bromide plus budesonide during the bronchospasm attacks.

Emergency physicians should be aware of pulmonary complications even with oral exposure to volatile liquids. Therefore, the occurrence of local pulmonary complications as well as the possible systemic complications should be closely monitored due to implication in pneumonitis but also in central nervous and gastrointestinal system toxicity, arrhythmias, hypokalemia and metabolic acidosis. The absence of symptoms or radiological findings immediately after thinner or any chemical ingestion or the presence of only short-lived symptoms is possibly determined by the amount and physical characteristics of the substance reaching tracheobronchial tree. Herewith, the healthcare professionals should be aware of developing serious complications in patients with chemical exposure who have normal physical findings initially. It should not be forgotten, in $5 \%$ of cases with pneumonitis, the disease progresses rapidly with severe manifestations such as multiple organ failure and ARDS (11).

\section{Conclusion}

The patients with a history of any chemical exposure should be hospitalized for at least twenty-four hours after exposure for monitoring of local and systemic toxic effects. It should be noted that the diagnosis of hydrocarbon exposure is based upon clinical features. It is not necessary to take the results of the laboratory tests rapidly because they do not change the management priorities.

\section{Ethics}

Informed Consent: It was taken.

Peer-review: Externally peer-reviewed.

\section{Authorship Contributions}

Concept: M.K., Design: M.K., Data Collection or Processing: M.K., K.A., Analysis or Interpretation: M.K., Literature Search: M.K., K.A., Writing: M.K.

Conflict of Interest: No conflict of interest was declared by the authors.

Financial Disclosure: The authors declared that this study received no financial support.

\section{References}

1. Malingre MM, Hendrix EA, Schellens JHM, Koks CHW, Tibben MM, Challa EE, et al. Acute poisoning after oral intake of a toluene-containing paint thinner. Eur J Pediatr. 2002;161:354-5.

2. Jayashree M, Singhi S, Gupta A. Predictors of outcome in children with hydrocarbon poisoning receiving intensive care. Indian Pediatr. 2006;43:7159.

3. Yang CC, Wu JF, Ong HC, Hung SC, Kuo YP, Sa CH, et al. Taiwan National Poison Center: epidemiologic data 1985-1993. J Toxicol Clin Toxicol. 1996;34:651-63.

4. Gurkan F, Bosnak M. Use of nebulized budesonide in two critical patients with hydrocarbon intoxication. Am J Ther. 2005;12:366-7.

5. Horoz 00, Yildizdas D, Yilmaz HL. Surfactant therapy in acute respiratory distress syndrome due to hydrocarbon aspiration. Singapore Med J. 2009;50:e130-2.

6. Ellenhorn MJ. The hydrocarbon products. In: Ellenhorn's Medical Toxicology: Diagnosis and Treatment of Human Poisoning, 2nd, Ellenhorn MJ, Schonwald S, Ordog G, Wasserberger (Eds), Williams \& Wilkins, Baltimore 1997. p.1420

7. Ameno K, Fuke C, Ameno S, Kiriu T, Sogo K, Ijiri I. A fatal case of oral ingestion of toluene. Forensic Sci Int. 1989;41:255-60.

8. Camara-Lemarroy CR, Rodríguez-Gutiérrez R, Monreal-Robles R, GonzálezGonzález JG. Acute toluene intoxication-clinical presentation, management and prognosis: a prospective observational study. BMC Emerg Med. 2015;15:19.

9. Rahimi HR, Agin K, Shadnia S, Hassanian-Moghaddam H, Oghazian MB. Clinical and biochemical analysis of acute paint thinner intoxication in adults: a retrospective descriptive study. Toxicol Mech Methods. 2015;25:427.

10. Steele RW, Conklin RH, Mark HM. Corticosteroids and Antibiotics for the Treatment of Fulminant Hydrocarbon Aspiration. JAMA J Am Med Assoc. 1972;219:1434

11. Makrygianni EA, Palamidou F, Kaditis AG. Respiratory Complications Following Hydrocarbon Aspiration in Children. Pediatr Pulmonol. 2016;51:560-9. 